\title{
Envelhecimento e Prática de Rejuvenescimento: Estudo de Representações Sociais
}

\author{
Adriana de Aguiar \\ Universidade Federal de Santa Catarina, SC, Brasil. $\quad$ Universidade Federal de Santa Catarina, SC, Brasil. \\ Andréa Barbará da Silva Bousfield \\ Universidade Federal de Santa Catarina, SC, Brasil.
}

\begin{abstract}
Resumo: Este estudo teve como objetivo identificar o conteúdo e a estrutura das representações sociais do envelhecimento e da prática de rejuvenescimento, a partir da abordagem estrutural das representações sociais. As participantes foram mulheres de meia-idade $(\mathrm{N}=30)$, com atitudes favoráveis e contrárias a práticas de rejuvenescimento, que responderam a duas redes associativas com as palavras-estímulo: envelhecimento e prática de rejuvenescimento, cujos dados foram submetidos à análise lexicográfica. Os resultados mostram que o núcleo central das representações sociais do envelhecimento é organizado em torno de perdas e ganhos, enquanto que o núcleo central das representações sociais da prática de rejuvenescimento remete a saúde, beleza e estados subjetivos. A saúde aparece como organizadora das representações sociais de ambos os objetos, ligando-se a elementos que remetem à esfera subjetiva, sobretudo entre as mulheres desfavoráveis a práticas de rejuvenescimento e elementos relacionados à dimensão corporal, entre as favoráveis. Conclui-se que as representações sociais do envelhecimento e da prática de rejuvenescimento são ancoradas na ideologia do envelhecimento ativo e bem-sucedido, em que o bom envelhecimento não é apenas desejável, mas se estabelece como uma norma a ser seguida.
\end{abstract}

Palavras-chave: Representações Sociais, Envelhecimento, Prática de Rejuvenescimento.

\section{Aging and Rejuvenation Practice: Study of Social Representations}

\begin{abstract}
This study aimed to identify the content and structure of social representations of aging and the practice of rejuvenation, from the structural approach of social representations. The participants were middle-aged women $(\mathrm{N}=30)$, with favorable and unfavorable attitudes to rejuvenation practices, who responded to two associative networks with the stimulus words: aging and rejuvenation practice. Data were then subjected to lexicographic analysis. The results show that the central nucleus of social representations of aging is organized around losses and gains, whereas the central nucleus of the social representations of the practice of rejuvenation refers to health, beauty and subjective states. Health appears as the organizer of social representations of both objects, linking to elements that refer to the subjective sphere, especially among women unfavorable to rejuvenation practices, and elements related to the body dimension among the favorable ones. It can be concluded that social representations of aging and the practice of rejuvenation are anchored in the ideology of active and successful aging, in which good aging is not only desirable but is established as a norm to be followed.
\end{abstract}

Keywords: Social Representations, Aging, Rejuvenation Practice. 


\title{
Envejecimiento y Práctica de Rejuvenecimiento: Estudio de Representaciones Sociales
}

\begin{abstract}
Resumen: Este estudio tuvo como objetivo identificar el contenido y la estructura de las representaciones sociales del envejecimiento y de la práctica de rejuvenecimiento, a partir del enfoque estructural de las representaciones sociales. Las participantes fueron mujeres de mediana edad $(\mathrm{N}=30)$, con actitudes favorables y contrarias a prácticas de rejuvenecimiento, que respondieron a dos redes asociativas con las palabras-estímulo: envejecimiento y práctica de rejuvenecimiento, cuyos datos fueron sometidos al análisis lexicográfico. Los resultados muestran que el núcleo central de las representaciones sociales del envejecimiento se organiza en torno a pérdidas y ganancias, mientras que el núcleo central de las representaciones sociales de la práctica de rejuvenecimiento remite a la salud, belleza y estados subjetivos. La salud aparece como organizadora de las representaciones sociales de ambos objetos, ligándose a elementos que remiten a la esfera subjetiva, sobre todo entre las mujeres desfavorables a prácticas de rejuvenecimiento y elementos relacionados a la dimensión corporal, entre las favorables. Se concluye que las representaciones sociales del envejecimiento y la práctica de rejuvenecimiento se anclan en la ideología del envejecimiento activo y exitoso, en el que el buen envejecimiento no es sólo deseable, sino que se establece como una norma a seguir.
\end{abstract}

Palabras clave: Representaciones Sociales, Envejecimiento, Práctica de Rejuvenecimiento.

\section{Introdução}

O envelhecimento é um processo natural e universal, portanto inerente a todos os seres vivos. No entanto, o avanço biotecnológico, associado à significativa melhoria na qualidade de vida tem permitido que as pessoas alcancem estágios de desenvolvimento cada vez mais avançados no ciclo de vida. No Brasil, dados do Instituto Brasileiro de Geografia e Estatística (2016) indicam que o perfil demográfico vem passando por intensas mudanças, tornando-se velozmente cada vez mais envelhecido.

O perfil populacional mais envelhecido em um país que atribui grande importância ao corpo e à beleza (Camargo, Goetz, Bousfield, \& Justo, 2011) tem impulsionado um intenso movimento no sentido de adiar ou tentar evitar o processo de envelhecimento, por meio de iniciativas que objetivam a manutenção da jovialidade física, seja no seu aspecto estético ou relativo à funcionalidade do corpo. Como as mulheres apresentam maior insatisfação com a imagem corporal (Miranda, Filgueiras, Neves, Teixeira, \& Ferreira, 2012) e são mais sensíveis às influências sociais em relação à aparência quando comparadas aos homens (Camargo, Justo, \& Jodelet, 2010), elas podem sofrer maior impacto em relação à visibilidade do envelhecimento.
Enquanto produto social e simbólico, o corpo é uma realidade culturalmente construída, modelado por forças históricas (Ferreira, 2013), o que faz com que objetos diretamente relacionados a ele, como o envelhecimento, sejam engendrados em valores, normas e crenças vigentes em um determinado contexto sociocultural. Ao mesmo tempo em que as pessoas rejeitam parecer ou ser enquadradas como "velhas" (Schneider, \& Irigaray, 2008), atualmente também se observa um movimento na sociedade de valorização da velhice, impulsionado pela difusão dos conceitos de envelhecimento ativo e bem-sucedido (Baltes \& Baltes, 1990; OMS, 2005), em que o envelhecer com qualidade de vida está relacionado ao equilíbrio entre perdas e ganhos na velhice (Baltes, 1987). Embora admitindo o declínio natural associado ao processo de envelhecer, essas concepções remetem à necessidade de ações visando o combate das perdas, em que a pressão social é direcionada a desejar "envelhecer de forma jovem" e "lutar contra o envelhecimento" (Caradec, 2011), o que aproxima as concepções de envelhecimento ativo e bem-sucedido da noção de antienvelhecimento (Flatt, Settersten Junior, Ponsaran, \& Fishman, 2013).

O desejo e a figura da juventude fazem parte do imaginário social e da cultura no Brasil (Arruda, 2012), tornando o antienvelhecimento uma ideologia ampla- 
mente difundida na sociedade, que alicerça saberes e práticas diversificadas, empregadas cotidianamente pelos indivíduos com intuito de promover o retardo do envelhecimento ou mesmo o rejuvenescimento físico. Portanto, o estudo do envelhecimento em Psicologia social, bem como o das ações de resistência ao mesmo, requer que se aborde o que as pessoas pensam sobre estes objetos, de forma que a teoria das representações sociais pode dar uma importante contribuição para atingir tal objetivo.

As representações sociais são teorias leigas, formadas com o objetivo de tornar familiares fatos desconhecidos, atribuindo-lhes um sentido que torne a realidade compreensível (Moscovici, 1978). Essas teorias são utilizadas pelos indivíduos como grades de interpretação da realidade e surgem da necessidade de ajustamento das pessoas aos objetos e eventos da vida cotidiana. Originam-se a partir de um processo criativo de elaboração cognitiva e simbólica que se dá no intercâmbio das relações e comunicações sociais (Nóbrega, 2003), e no qual os processos comunicativos se constituem no veículo que promove sua gênese e difusão (Moscovici, 1978). Existem diferentes abordagens de estudo das representações sociais, cada uma delas avaliando o processo de construção desta forma de conhecimento com enfoques distintos e complementares: 1) a abordagem dimensional (Moscovici, 1978) se atém principalmente a descrever como as representações sociais são construídas e distingue três dimensões - informação, atitudes e campo; 2) a abordagem dinâmica (Jodelet, 1989) compreende as representações sociais sob uma perspectiva prática, na medida em que propõe que elas são construídas em decorrência de uma necessidade das pessoas de saber como agir no cotidiano; 3) a abordagem genética (Doise, 1985) dá maior ênfase ao processo de ancoragem e busca encontrar o princípio organizador das representações sociais; 4) a abordagem dialógica (Marková, 2003) enfatiza a natureza interativa e dinâmica das representações sociais, concebendo-as como formas simbólicas resultantes da relação triádica entre um Ego, um Alter e um objeto; e 5) a abordagem estrutural (Abric, 1998; 2003) concentra a atenção em como se estruturam os conteúdos representacionais ligados ao objeto, e é a abordagem enfatizada neste estudo.

Segundo a abordagem estrutural, as representações sociais estruturam-se em torno de dois sistemas de cognições complementares: um núcleo central, composto por elementos mais estáveis e que tem como função organizar e dar estabilidade à representação, e um sistema periférico, composto por elementos mais flexíveis e concretos, que se alteram com mais facilidade em decorrência do contexto social dos indivíduos (Abric, 2003; Flament, 2001). A determinação do sistema central é social, ligada às condições históricas, sociológicas e ideológicas, portanto a normas e valores. O núcleo central tem uma função geradora $\mathrm{e}$ estruturante, na medida em que dá significado e estabilidade à representação social, determinando a natureza dos elos entre os elementos que a compõe. Já o sistema periférico apresenta os elementos mais acessíveis e concretos da representação e é mais associado às características individuais e ao contexto imediato no qual os indivíduos estão inseridos (Abric, 1998).

Pesquisas sobre as representações sociais do envelhecimento tem revelado que o conhecimento sobre este objeto é organizado em torno da dicotomia corpo $\mathrm{X}$ mente, remetendo a um contraste entre perdas corporais, como da saúde e beleza, e ganhos subjetivos, tais como sabedoria, maturidade e experiência (Camargo, Contarello, Wachelke, Morais, \& Piccolo, 2014; Schulze, 2011; Torres, Camargo, Bousfield, \& Silva, 2015). Outros estudos relacionam o envelhecimento a estados subjetivos como a manutenção de um "espírito jovem" e exercício de práticas consideradas "não velhas" (Biasus, Demantova, \& Camargo, 2011; Cruz, \& Ferreira, 2011; Magnabosco-Martins, Camargo \& Biasus, 2009), remetendo a ideias relativas ao rejuvenescimento.

Diferentemente do que ocorre com o envelhecimento, os estudos sobre as representações sociais do rejuvenescimento ainda são incipientes, e tem situado a ancoragem deste objeto em três domínios: como um objeto de saúde, ligado à preservação da funcionalidade corporal e ao poder de autonomia, como um objeto de beleza, atrelado a práticas estéticas para a manutenção da jovialidade física, e como um objeto ligado a uma dimensão subjetiva, que remete à jovialidade mental, ao bem-estar e a emoções positivas (Aguiar, 2016; Berri, Castro, \& Camargo, 2016; Castro, Antunes, Brito, \& Camargo, 2016; Torres, 2010).

O conhecimento das representações sociais do envelhecimento e da prática de rejuvenescimento possibilita entender como as pessoas pensam questões relativas à velhice, e como este pensamento sustenta práticas sociais relacionadas ao seu enfrentamento. Como o pensamento característico das sociedades modernas é plural (Jovchelovitch, 2011), é esperado que coexistam, mesmo dentro de um mesmo grupo 
ou categoria social, diferentes formas de saber sobre o envelhecimento e o rejuvenescimento. Embora as representações sociais sejam formadas pelas dimensões informação, campo e atitude (Moscovici, 1978), autores atribuem um papel de destaque a esta última dimensão, considerando as atitudes como elementos primários na formação e organização das cognições que compõe o campo representacional associada ao objeto (Parales-Quenza, \& Vizicaíno-Gutiérrez, 2007). Desta forma, é possível supor que diferentes atitudes frente a práticas de rejuvenescimento estejam organizadas em torno de diferentes representações tanto sobre este objeto quanto sobre o envelhecimento. Este estudo teve como objetivo identificar o conteúdo e como se estruturam as representações sociais do envelhecimento e de práticas de rejuvenescimento para mulheres com atitudes positivas e negativas em relação a este último objeto.

\section{Método}

\section{Participantes}

Participaram deste estudo 30 mulheres com idade entre 38 e 62 anos $(M=51$ anos e 4 meses; $D P=$ 7 anos e 8 meses), 15 com atitudes positivas, consideradas favoráveis a práticas de rejuvenescimento, e 15 com atitudes negativas, consideradas desfavoráveis. As atitudes foram identificadas a partir de oito práticas de rejuvenescimento (alimentação voltada ao rejuvenescimento, exercícios físicos, cosméticos, tinturas para cabelo, toxina botulínica, peeling químico, preenchimento cutâneo e cirurgia plástica) e foram mensuradas em um estudo anterior (Aguiar, 2016).

\section{Instrumento}

As representações sociais foram investigadas por meio de duas redes associativas (De Rosa, 2005), uma utilizando a palavra "envelhecimento" como termo indutor e outra o termo "prática de rejuvenescimento". Foi solicitado às participantes que evocassem todas as palavras referentes a cada palavra estímulo que lhe viessem à mente, identificando a ordem de evocação e de importância das palavras e atribuindo às mesmas uma valência positiva, negativa ou neutra. A ordem de evocação é utilizada como um indicador de saliência e acessibilidade prototípica dos elementos ao estímulo apresentado, enquanto que a ordem de importância implica em um processo cognitivo de ordem mais racional, envolvendo uma tarefa de caráter avaliativo.
As valências atribuídas tiveram como objetivo identificar os índices de polaridade, que se constituem em uma medida sintética de avaliação e atitude implícita no campo representacional (De Rosa, 2005).

\section{Procedimentos e aspectos éticos}

As participantes foram recrutadas a partir de um estudo anterior (Aguiar, 2016). A pesquisa cumpriu todas as obrigações éticas, obtendo aprovação do Comitê de Ética em Pesquisas com Seres Humanos da Universidade Federal de Santa Catarina (parecer consubstanciado $n^{\circ} 370.109 / 2013$ ).

\section{Análise de dados}

O material textual foi analisado de duas formas: primeiramente foi calculado o poder indutivo, que apresenta as médias de palavras evocadas pelas participantes, e o índice de polaridade, que varia de $-1 \mathrm{a}+1$ (De Rosa, 2005). Para tal foram utilizadas as seguintes fórmulas:

$$
\text { Poder Indutivo }(\mathrm{PI})=\frac{\mathrm{n}^{\mathrm{o}} \text { de palavras }}{\mathrm{n}^{\mathrm{o}} \text { total de participantes }}
$$

Índice de polaridade $(\mathrm{IP})=\frac{\mathrm{n}^{\mathrm{o}} \text { de palavras positivas }-\mathrm{n}^{\mathrm{o}} \text { de palavras negativas }}{\mathrm{n}^{\mathrm{o}} \text { total de palavras associadas }}$

As diferenças de médias entre os dois grupos de participantes foram verificadas por meio de estatística não paramétrica (teste Mann-Whitney). Após, o material textual foi submetido a uma caracterização estrutural, visando identificar o núcleo central e periférico, utilizando-se o programa informático EEvocation (Ensemble de programmes permettant l'analyse des evocations) (versão 2005). A análise efetuada por este programa baseia-se na tabulação cruzada de dois critérios: a frequência de aparecimento da palavra e sua ordem média de evocação (Vergès, 1992). No entanto, neste estudo optou-se por realizar também a análise considerando como critério a ordem de importância atribuída à palavra, pois a complementariedade de ambos os critérios permite identificar com maior rigor os campos da representação social ao revelar seu significado associativo e funcional (Dany, Urdapilleta, \& Lo Monaco, 2015).

A estrutura das representações sociais, na qual se buscou verificar as conectividades entre seus elementos, foi identificada por meio de análise de similitude, com o auxílio do software IRaMuTeQ (Interface de R pour les Analyses Multidimensionnelles de Textes et de Questionnaires) (versão 0.7 alfa 2) (Camargo, \& 
Justo, 2013). As palavras foram categorizadas, agrupando-se na mesma categoria as que apresentassem sentidos semelhantes.

\section{Resultados}

\section{Termo indutor: envelhecimento}

Em relação ao termo indutor Envelhecimento, obteve-se um total de 341 evocações, com 172 palavras diferentes (média de 11 evocações por participante entre favoráveis e 12 entre as desfavoráveis às práticas de rejuvenescimento). Verificou-se polaridade positiva entre as participantes desfavoráveis $(M=0,64$, $D P=0,36)$ e polaridade ligeiramente negativa entre as participantes favoráveis $(M=-0,03 ; D P=0,42)$, o que indica uma atitude muito positiva em relação ao envelhecimento por parte das primeiras e negativa por parte das segundas, embora neste último caso com tendência à neutralidade $(U=24, Z=-3,68, p<0,001)$.

Quanto ao conteúdo das representações sociais, as palavras evocadas foram analisadas considerando a combinação da frequência média de evocação $(f)$ com ordem média de evocação (OME) e frequência média de evocação a ordem média de importância (OMI). O quadrante superior e à esquerda da tabela (Tabela 1), apresenta os elementos mais frequentes e mais prontamente evocados (<OME) e são indicativos do núcleo central da representação social. O quadrante superior e à direita constitui a primeira periferia e traz elementos importantes devido a sua frequência, embora com OME maior. $\mathrm{O}$ quadrante inferior e à esquerda constitui a zona de contraste, pois traz elementos pouco frequentes, mas que estavam entre os primeiros a serem evocados. O quadrante inferior e à direita corresponde à segunda periferia, com os elementos menos frequentes e evocados por último. Como se trata de um estudo de representações sociais, foram desprezadas as palavras com frequência igual a 1, o que correspondeu a 33,7\% das evocações.

Considerando-se a OME (Tabela 1 - quadrantes superiores), no primeiro quadrante destacam-se elementos que evidenciam uma representação do envelhecimento focada nas perdas (rugas, solidão e limitações) e ganhos (sabedoria, maturidade, experiência, liberdade e conhecimento) obtidos ao longo do tempo, bem como um processo intrínseco ao ser humano (natural) e que envolve uma preocupação com o corpo e a funcionalidade (saúde e exercícios físicos). O contraste entre perdas e ganhos também é evidenciado na periferia da representação, assim como as estratégias para manter o bem-estar físico e subjetivo, além da proximidade com a morte.

Ao se considerar a OMI (Tabela 1 - quadrantes inferiores), observam-se mudanças em apenas três elementos no possível núcleo central, o que demonstra que, em geral, os elementos mais evocados são também considerados os mais importantes. Nota-se que a centralidade da representação passa e englobar predominantemente elementos positivos, com elementos negativos tornando-se periféricos (rugas, solidão e limitações) e positivos tornando-se centrais (família, alegria e serenidade).

Para visualizar como se estrutura a representação social do envelhecimento e confirmar a centralidade dos seus elementos, foi realizada uma análise de co-ocorrência com base em categorias desenvolvidas a partir das palavras evocadas. Para a construção da árvore máxima, considerando-se o reduzido número de participantes, utilizou-se o filtro entre as categorias de palavras de um mínimo de cinco co-ocorrências (Figura 1).

Como pode ser observado na Figura 1, o elemento saúde organiza a representação social deste objeto, apresentando forte conexão com o elemento maturidade e este com os cuidados que se deve ter no envelhecimento. A saúde também aparece conectada aos sinais físicos do envelhecimento, a experiência, a serenidade e aos exercícios físicos. A serenidade, por sua vez, conecta-se à sabedoria, que se mostra relacionada com aspectos subjetivos do envelhecimento, como a liberdade e alegria. A ligação entre esses elementos mais subjetivos foi destacada pelas participantes desfavoráveis, sugerindo uma representação social organizada predominantemente ao redor dos ganhos do envelhecimento. Por outro lado, a ligação entre saúde, maturidade e sinais físicos foi mais saliente para as participantes favoráveis, o que sugere uma representação social organizada em torno de um contraste entre ganhos subjetivos e perdas físicas, enfatizando os problemas de saúde e mudanças na aparência. Nota-se também que os sinais físicos aparecem conectados à solidão e esta, ao elemento medo, o que denota que o declínio físico provocado pelo envelhecimento se relaciona ao declínio social, o que se constitui como um aspecto ameaçador para as mulheres de ambos os grupos. Assim, a conexão entre os elementos mostrada pela análise de similitude confirma a centralidade dos mesmos na representação social do envelhecimento. 
Tabela 1

Quadrantes referentes as representações sociais do envelhecimento segundo critérios de OME e OMI.

\begin{tabular}{|c|c|c|c|c|c|c|}
\hline & \multicolumn{6}{|c|}{ Critério 1: Ordem de Evocação } \\
\hline & \multicolumn{3}{|c|}{$\mathrm{OME}<7$} & \multicolumn{3}{|c|}{$\mathrm{OME} \geq 7$} \\
\hline & Palavra & $\mathrm{F}$ & OME & Palavra & $\mathrm{F}$ & OME \\
\hline \multirow{12}{*}{$\begin{array}{l}L 0 \\
\wedge \\
\Lambda \\
L\end{array}$} & sabedoria & 14 & 4,07 & família & 10 & 7,30 \\
\hline & maturidade & 13 & 6,15 & morte & 10 & 8,00 \\
\hline & saúde & 12 & 5,50 & alegria & 8 & 8,50 \\
\hline & rugas & 11 & 5,54 & serenidade & 7 & 7,14 \\
\hline & experiência & 10 & 3,90 & cuidados & 6 & 7,17 \\
\hline & solidão & 10 & 6,10 & natureza & 6 & 8,50 \\
\hline & tempo & 8 & 5,87 & medo & 5 & 11,00 \\
\hline & liberdade & 8 & 6,12 & & & \\
\hline & natural & 6 & 1,33 & & & \\
\hline & limitações & 6 & 6,00 & & & \\
\hline & conhecimento & 5 & 3,40 & & & \\
\hline & exercícios físicos & 5 & 6,80 & & & \\
\hline \multirow{7}{*}{$\begin{array}{l}L \\
v \\
1\end{array}$} & responsabilidade & 4 & 5,50 & dor & 4 & 7,00 \\
\hline & dificuldades & 4 & 6,50 & paciência & 4 & 7,50 \\
\hline & & & & enfraquecimento & 4 & 8,00 \\
\hline & & & & perdas & 4 & 8,75 \\
\hline & \multicolumn{6}{|c|}{ Critério 2: Ordem de Importância } \\
\hline & \multicolumn{3}{|c|}{$\mathrm{OMI}<7$} & \multicolumn{3}{|c|}{$\mathrm{OMI} \geq 7$} \\
\hline & Palavra & $\mathrm{F}$ & OMI & Palavra & $\mathrm{F}$ & OMI \\
\hline \multirow{11}{*}{$\begin{array}{l}\stackrel{L}{1} \\
\mathrm{~N}_{W}\end{array}$} & sabedoria & 14 & 3,71 & rugas & 11 & 10,00 \\
\hline & maturidade & 13 & 6,61 & solidão & 10 & 10,20 \\
\hline & saúde & 12 & 2,91 & morte & 10 & 8,55 \\
\hline & família & 10 & 5,10 & limitações & 6 & 7,16 \\
\hline & experiência & 10 & 6,10 & cuidados & 6 & 7,17 \\
\hline & liberdade & 8 & 3,00 & natural & 6 & 7,83 \\
\hline & tempo & 8 & 5,75 & natureza & 6 & 8,50 \\
\hline & alegria & 8 & 6,50 & medo & 5 & 13,00 \\
\hline & serenidade & 7 & 6,28 & & & \\
\hline & exercícios físicos & 5 & 6,20 & & & \\
\hline & conhecimento & 5 & 3,60 & & & \\
\hline \multirow{3}{*}{$\begin{array}{l}10 \\
v \\
1\end{array}$} & responsabilidades & 4 & 4,75 & dor & & 7,75 \\
\hline & paciência & 4 & 5,25 & perdas & & 9,50 \\
\hline & & & & enfraquecimento & & 11,50 \\
\hline
\end{tabular}

\section{Termo indutor: Prática de rejuvenescimento}

Com o termo indutor Prática de Rejuvenescimento, foram obtidas 278 evocações, com 118 palavras diferentes e uma média de aproximadamente nove evocações por participante, verificando-se este padrão entre as participantes dos dois posicionamentos. Quanto ao índice de polaridade, tanto as participantes favoráveis $(M=0,67 ; D P=0,40)$ quanto desfavoráveis $(M=0,43$; $\mathrm{DP}=0,68)$ apresentaram polaridade positiva, sem diferenças significativas entre as médias.
Quanto a estrutura da representação social da prática de rejuvenescimento, os critérios adotados para a escolha do vocabulário foram os mesmos apresentados anteriormente, desprezando-se $28,4 \%$ das palavras com frequência igual a 1 (Tabela 2).

Em relação à OME (Tabela 2 - quadrantes superiores), baseando-se no primeiro quadrante, evidencia-se uma representação que liga práticas de rejuvenescimento a três dimensões: saúde (exercícios físicos e alimentação), beleza (cirurgia plástica, cosméticos e custo) e subjetiva (alegria e 


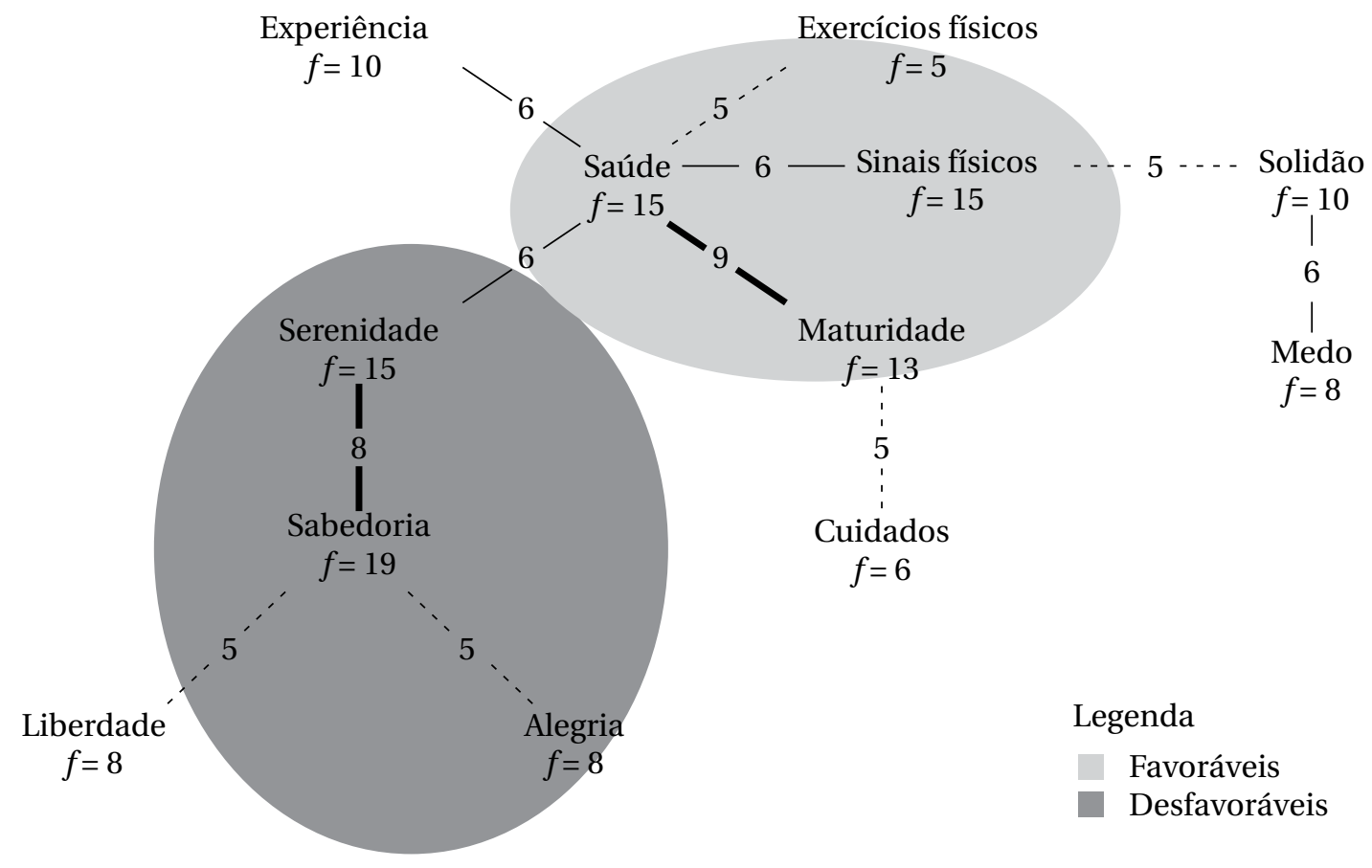

Figura 1

Representação gráfica da árvore máxima das representações sociais do envelhecimento.

autoestima). Na periferia da representação social, verifica-se ênfase em elementos alusivos ao rejuvenescimento enquanto busca da beleza e sob uma perspectiva subjetiva, em que a leitura se constitui uma estratégia para a manutenção da atividade intelectual e juventude mental.

Ao se considerar a OMI (Tabela 2 - quadrantes inferiores), notam-se algumas diferenças, em comparação com a OME, na distribuição dos elementos nos quadrantes. $\mathrm{O}$ elemento cirurgia plástica, um dos mais frequentes e primeiramente evocados, é indicado como o menos importante, deixando de ser um aspecto central na representação. Por outro lado, elementos pouco evocados, mas com a atribuição de grande importância, tais como paz e amor, tornaram-se centrais, o que, aliado à permanência dos elementos alegria e autoestima, indica uma alta valorização de aspectos mentais e afetivos na representação social da prática de rejuvenescimento. Desta forma, os recursos estéticos mais intervencionistas, embora salientes no imaginário social em torno das práticas de rejuvenescimento, são renegados a uma posição periférica em termos de importância, visto serem desvalorizados quando comparados às práticas de cuidado não invasivas, sejam elas estéticas, de saúde ou mesmo de caráter subjetivo.
Visando identificar a estrutura da representação social, utilizou-se, para a construção da árvore máxima, o filtro entre as categorias de palavras de um mínimo de seis co-ocorrências, como demonstra a Figura 2.

Observa-se, na Figura 2, que o elemento exercícios físicos organiza os demais, no entanto, mostra-se conectado com elementos referentes a práticas corporais voltadas a saúde e beleza entre as mulheres favoráveis e a saúde e bem-estar subjetivo entre as mulheres desfavoráveis. Além dos exercícios físicos, a alimentação foi um elemento presente na estrutura da representação social de ambos os grupos de mulheres, o que evidencia a primazia da saúde como ponto de interface entre as representações sociais dos dois objetos deste estudo: envelhecimento e prática de rejuvenescimento. No entanto, a conexão exercícios físicos - cirurgia plástica - tratamentos estéticos demonstra que as mulheres favoráveis consideram que a atividade física também pode contribuir para a beleza física e a aquisição de uma aparência mais jovial, quando associada a essas estratégias corporais estéticas. Por outro lado, entre as mulheres desfavoráveis, os exercícios físicos aparecem conectados também a elementos subjetivos, evidenciando que, quando associados à atividade mental, ao bom humor e uma postura serena perante a vida, contribuem para 
Tabela 2

Quadrantes referentes às representações sociais da prática de rejuvenescimento segundo critérios de OME e OMI.

\begin{tabular}{|c|c|c|c|c|c|c|}
\hline & \multicolumn{6}{|c|}{ Critério 1: Ordem de Evocação } \\
\hline & \multicolumn{3}{|c|}{$\mathrm{OME}<6$} & \multicolumn{3}{|c|}{$\mathrm{OME} \geq 6$} \\
\hline & Palavra & $\mathrm{F}$ & OME & Palavra & $\mathrm{F}$ & OME \\
\hline \multirow{8}{*}{$\begin{array}{l}\stackrel{L}{1} \\
\stackrel{4}{W}\end{array}$} & exercícios físicos & 21 & 3,28 & Tratamentos estéticos & 14 & 6,21 \\
\hline & alimentação & 15 & 2,40 & amizade & 8 & 6,75 \\
\hline & cirurgia plástica & 14 & 5,21 & leituras & 6 & 6,66 \\
\hline & cosméticos & 12 & 5,66 & paz & 6 & 7,50 \\
\hline & saúde & 10 & 4,80 & amor & 5 & 6,80 \\
\hline & alegria & 9 & 5,89 & & & \\
\hline & autoestima & 8 & 3,62 & & & \\
\hline & custo & 6 & 5,16 & & & \\
\hline \multirow{8}{*}{$\begin{array}{l}\stackrel{0}{v} \\
\mathrm{v} \\
+\end{array}$} & vaidade & 4 & 5,25 & yoga & 4 & 6,00 \\
\hline & & & & prazer & 4 & 7,50 \\
\hline & & & & viagens & 4 & 8,25 \\
\hline & & & & sexo & 4 & 9,75 \\
\hline & & & & sorrir & 4 & 11,75 \\
\hline & \multicolumn{6}{|c|}{ Critério 2: Ordem média de importância } \\
\hline & & & & & & \\
\hline & Palavra & $\mathrm{F}$ & OMI & Palavra & $\mathrm{F}$ & OMI \\
\hline \multirow{10}{*}{$\begin{array}{l}10 \\
\stackrel{1}{4}\end{array}$} & exercícios físicos & 21 & 5,04 & tratamentos estéticos & 14 & 8,01 \\
\hline & alimentação & 15 & 1,73 & cirurgia plástica & 14 & 11,0 \\
\hline & cosméticos & 12 & 5,91 & leituras & 6 & 6,00 \\
\hline & saúde & 10 & 3,70 & & & \\
\hline & alegria & 9 & 3,78 & & & \\
\hline & amizade & 8 & 5,37 & & & \\
\hline & autoestima & 8 & 3,50 & & & \\
\hline & paz & 6 & 5,17 & & & \\
\hline & custo & 6 & 5,50 & & & \\
\hline & amor & 5 & 5,00 & & & \\
\hline \multirow{5}{*}{$\begin{array}{l}10 \\
v \\
4\end{array}$} & yoga & 4 & 4,75 & vaidade & 4 & 6,25 \\
\hline & & & & prazer & 4 & 7,50 \\
\hline & & & & viagens & & 8,25 \\
\hline & & & & sorrir & & 8,75 \\
\hline & & & & sexo & & 8,75 \\
\hline
\end{tabular}

o rejuvenescimento mental. Assim, evidencia-se uma relação entre corpo e mente, em que o rejuvenescimento se atrela a ideia de um cuidado integral, que envolve as várias esferas da vida do indivíduo.

\section{Discussão}

Os resultados deste estudo confirmaram os dados da literatura, ao identificarem uma representação social do envelhecimento organizada em torno das perdas e ganhos deste processo (Camargo et al., 2014; Schulze, 2011; Torres et al., 2015). A análise das evocações oriundas da rede associativa de palavras con- firmou a centralidade da representação em torno de estereótipos etários positivos, de elementos pragmáticos referentes à qualidade de vida e ao declínio físico e social, resultados que têm sido recorrentes na literatura (Magnabosco-Martins et al., 2009; Schulze, 2011).

Ao se realizar uma comparação entre a ordem de evocação e de importância, observou-se diferentes padrões em termos de dinâmica centralidade-periferia envolvendo os elementos do núcleo central. Ao se considerar as palavras prontamente evocadas, os elementos solidão, rugas e limitações se mostram centrais, no entanto, em termos de importância, assumem características periféricas, cedendo lugar a elementos 


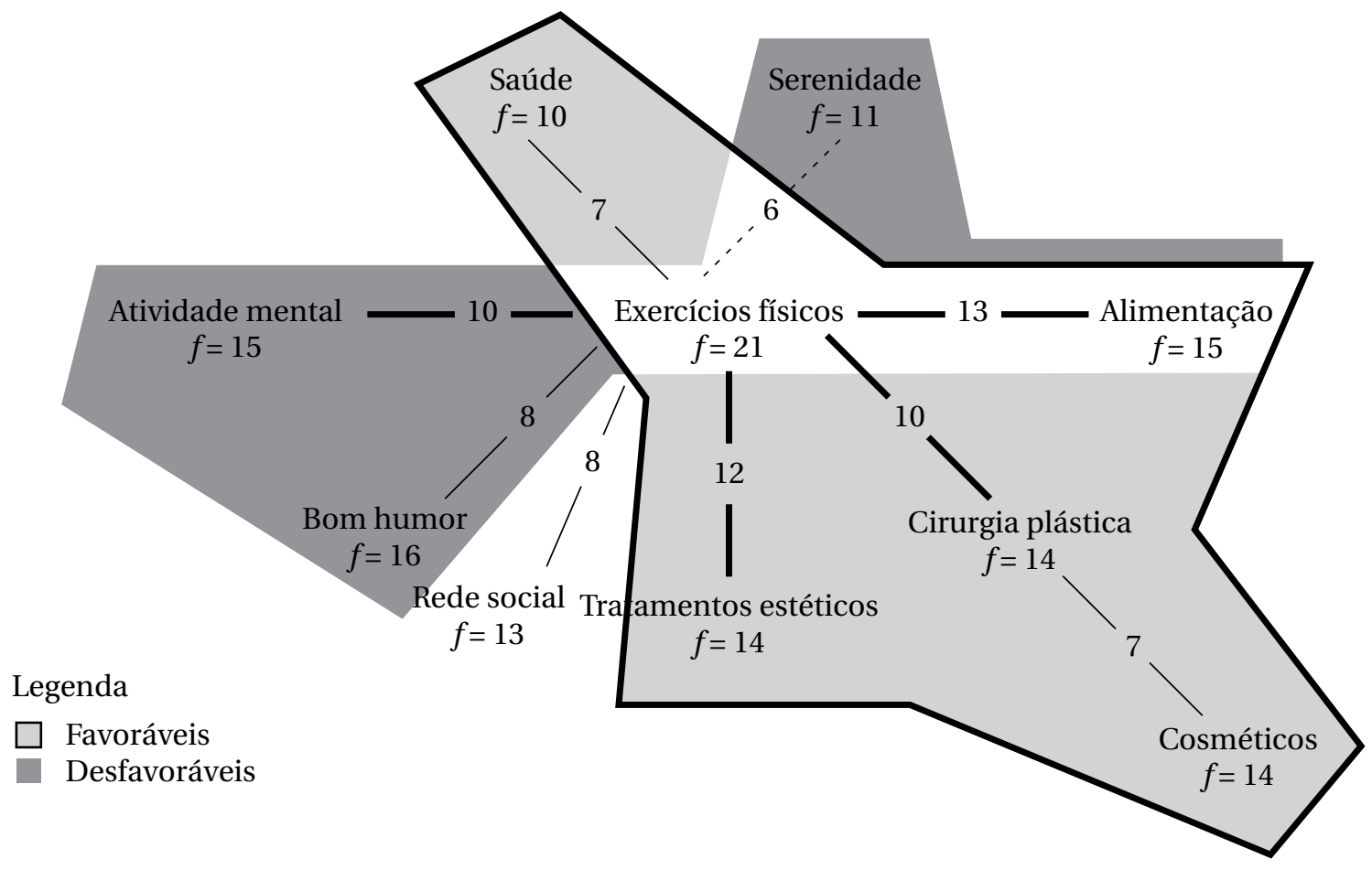

Figura 2

Representação gráfica da árvore máxima da representação social do envelhecimento.

positivos anteriormente periféricos, como família, alegria e serenidade. Por outro lado, o único elemento que alude a aspectos negativos que permanece central em termos de importância é saúde. Ao que parece, embora se identifique uma racionalidade prototípica centrada em torno das perdas e ganhos, em termos avaliativos predominam aspectos positivos do envelhecimento. Dany et al. (2015) defendem que as mudanças de centralidade dos elementos que podem ocorrer ao se considerar a ordem de evocação e importância acontecem devido a um processo de renegociação dos domínios do campo representacional; a operação de alocação de significado resultante não aconteceria ao acaso, mas seria governada por uma lógica psicossocial. Desta forma, é possível que as perdas do envelhecimento acionariam reações emotivas nas mulheres, cuja mudança do núcleo central viabilizaria uma minimização de uma dimensão ansiogênica da representação, acionada através dos elementos alusivos ao declínio físico e social, condições temidas e renegadas por serem socialmente desvalorizadas. A família, por sua vez, é considerada importante por se caracterizar como fonte de apoio no envelhecimento, enquanto que a alegria e serenidade correspondem a aquisições que contribuem para um melhor enfrentamento deste processo.
Ao se analisar a estrutura da representação, a saúde aparece como elemento organizador da representação social do envelhecimento, ligando-se a aquisições na esfera subjetiva, como a maturidade, serenidade e experiência, aos sinais físicos e aos exercícios físicos. O envelhecimento é representado em termos de um contraste entre ganhos subjetivos e perdas físicas, relacionadas tanto à saúde quanto à aparência, mas que podem ser minimizadas através da prática de exercícios físicos. A organização simbólica em torno de aquisições subjetivas é enfatizada pelas mulheres desfavoráveis às práticas de rejuvenescimento, enquanto que a estruturação em torno das perdas estéticas e funcionais e a obtenção de maturidade é salientada pelas favoráveis, o que evidencia representações ancoradas em domínios distintos: a primeira na concepção de mente e a segunda na de corpo.

Quanto ao índice de polaridade, que se constitui em uma medida sintética de avaliação e atitude implícita no campo representacional (De Rosa, 2005), os resultados mostraram que o envelhecimento é avaliado positivamente pelas participantes desfavoráveis, enquanto que as participantes favoráveis o avaliam de forma ligeiramente negativa, mas com tendência à neutralidade. Uma avaliação mais positiva por 
parte das desfavoráveis poderia decorrer em virtude de um campo representacional organizado em torno de elementos subjetivos que representariam ganhos do envelhecimento, enquanto que as favoráveis, além desses elementos, também considerariam questões corporais, que seriam relacionadas a perdas. As proposições do modelo Life-Span (Baltes, 1987) salientam que o equilíbrio entre as perdas e ganhos ao longo do desenvolvimento ocorrem devido a um processo compensatório, o que implica em considerar que um bom envelhecimento dependeria de uma compensação das perdas, associada a uma exploração das reservas em cada etapa da vida. Atitudes mais positivas entre as mulheres desfavoráveis indicam que o balanço entre perdas e ganhos resulta em um saldo positivo para essas participantes, enquanto que este balanço se mostra mais equilibrado entre as mulheres favoráveis.

Assim como observado com o objeto "envelhecimento", os elementos representacionais de "práticas de rejuvenescimento" também se organizaram em torno da díade corpo-mente. Quanto ao corpo, destacam-se elementos funcionais e pragmáticos que remetem a ações de combate ao envelhecimento corporal, enquanto que, no que se refere aos aspectos relativos à mente, os elementos remetem ao rejuvenescimento em uma perspectiva subjetiva, caracterizando-se como certos padrões de pensamento, sentimentos e de formas de se relacionar, resultados também encontrados por Torres (2010).

A representação social do objeto prática de rejuvenescimento parece se estruturar em torno de exercícios físicos, elemento que, por sua vez, está situado em campos representacionais distintos de acordo com a categoria de pertença das participantes. Entre as mulheres favoráveis a práticas de rejuvenescimento, este elemento se conecta a elementos relacionados a recursos estéticos e de saúde, o que sugere uma representação em que rejuvenescer implica em enfrentar os efeitos físicos do envelhecimento em sua integralidade, envolvendo saúde e aparência. Assim, a beleza é considerada um aspecto importante de ser saudável (Brooks, 2010), o que denota a feiura como uma moléstia associada ao envelhecimento, evidenciando representações sociais impregnadas de uma racionalidade biomédica. Por outro lado, entre as mulheres desfavoráveis, a estrutura da representação social de rejuvenescimento mostra que os exercícios físicos compõe um campo representacional cuja corporiedade é menos enfatizada, ligando-a também à alimen- tação, mas sobretudo a elementos subjetivos e afetivos, como a serenidade, o bom humor e atividade mental. Juntamente com a saúde física, esses fatores são muito valorizados por essas mulheres, o que foi evidenciado pela polaridade positiva identificada na rede associativa, cujas palavras remeterem predominantemente à faceta subjetiva e funcional do rejuvenescimento.

Quanto à dinâmica centralidade-periferia da representação social da prática de rejuvenescimento, a mudança de núcleo central ao se considerar a ordem de importância, principalmente em relação ao elemento cirurgia plástica, sugere certa resistência das participantes em considerar a beleza como um dos aspectos mais importantes associados ao objeto, o que pode indicar que o conhecimento sobre este objeto seja atravessado por questões de desejabilidade social. Embora o Brasil se destaque mundialmente na adesão à cirurgia plástica, segundo dados da International Society of Aesthetic Plastic Surgery (2014), este recurso estético também é considerado arriscado e associado à falta de limites e à futilidade (Aguiar, 2016; Castro et al., 2016). Desta forma, a desvalorização da cirurgia plástica poderia refletir um esforço das mulheres no sentido de evitar serem socialmente estigmatizadas.

Os resultados mostram que a saúde aparece como ponto de interface entre as representações sociais dos dois objetos abordados neste estudo, o que sugere uma ancoragem das representações sociais da prática de rejuvenescimento nas representações sociais do envelhecimento. A centralidade da saúde para ambas as representações ilustra a dimensão funcional e normativa do núcleo representacional (Abric, 1994), uma vez que revela visões de mundo impregnadas pela ideologia do envelhecimento ativo e bem-sucedido (Baltes, \& Baltes, 1990; OMS, 2005) e evidencia valores e normas que circulam na sociedade, em que envelhecer bem é interpretado como um imperativo moral, e pode ser obtido por meio de determinadas iniciativas, associadas a práticas de rejuvenescimento. Contudo, os dados sugerem que o bom envelhecimento não se limita à esfera funcional, já que também se refere à manutenção de uma "boa aparência", enfatizada principalmente pelas mulheres favoráveis a práticas de rejuvenescimento, ou mesmo de uma jovialidade mental, aspecto salientado na representação social das mulheres desfavoráveis a estas práticas. Considerando que a proximidade com o objeto social torna o núcleo da representação social mais funcional (Abric, 2003), é possível justificar uma maior saliência do 
rejuvenescimento saúde-beleza para as participantes favoráveis e o de saúde-mente para as desfavoráveis a práticas de rejuvenescimento.

\section{Considerações finais}

O objetivo deste estudo foi investigar o conteúdo e a estrutura das representações sociais do envelhecimento e da prática de rejuvenescimento em mulheres com atitudes antagônicas em relação a este último objeto. As representações sociais do envelhecimento organizaram-se em torno de perdas e ganhos, enquanto a da prática de rejuvenescimento em torno de elementos pragmáticos direcionados a estratégias de combate a essas perdas e conquista de um envelhecimento ativo e bem-sucedido; este parece envolver, além de uma dimensão funcional, também uma dimensão estética e uma mental. O núcleo central da representação social do envelhecimento evidencia estereótipos em relação a este objeto, bem como sistemas normativos que ilustram a desejabilidade social em relação aos cuidados com um corpo que, inexoravelmente, se deteriora com o tempo. Estes cuidados remetem a concepções de rejuvenescimento, de forma que a representação sobre este objeto se mostra ancorada na representação social do envelhecimento. Os dados sugerem uma saliência da dimensão corporal para as mulheres favoráveis a práticas de rejuvenescimento, enquanto os elementos representacionais das desfavoráveis acionam uma dimensão corporal-subjetiva.

Como limitações deste estudo, destaca-se o número reduzido de participantes e consequentemente de evocações, o que representou uma limitação à análise, de forma que a interpretação dos resultados deve ser relativizada e considerada em conjunto com os resultados de outros estudos. Outro ponto refere-se à amostra de conveniência, que limita a capacidade de generalização dos resultados. Desta forma, outros estudos com amostras representativas precisam ser desenvolvidos a fim de oferecer maior suporte empírico aos achados desta pesquisa e que incluam comparações geracionais, o que também não foi realizado neste estudo.

\section{Referências}

Abric, J.-C. (1998). A abordagem estrutural das representações sociais. In A. S. P. Moreira \& D. C. Oliveira, Estudos interdisciplinares de representação social (pp. 27-38). Goiânia, GO: AB.

Abric, J.-C. (2003). Abordagem estrutural das representações sociais: desenvolvimentos recentes. In P. H. F. Campos \& M. C. S. Loureiro (Orgs.), Representações sociais e práticas educativas (pp. 37-57). Goiânia, GO: UCG.

Abric, J-C.(1994). Introduction. In J.-C. Abric (Org.), Pratiques sociales et représentations (pp.07-09). Paris: PressesUniversitaires de France.

Aguiar, A. (2016). Envelhecimento e rejuvenescimento em contextos intergrupais antagônicos: representações sociais e práticas corporais (Tese de Doutorado) Universidade Federal de Santa Catarina, Florianópolis.

Arruda, A. (2012) Envelhecer: uma novidade? In L.F. R. Tura, \& A. O. Silva (Orgs), Envelhecimento e representações sociais (pp. 19-33). Rio de Janeiro, RJ: Quartet Fapery.

Baltes, P. B. (1987). Theorical propositions of lifespan developmental psychology on the dynamics between growth and decline. Developmental Psychology, 23(5), 611-626. https://doi.org/10.1037/a0021500

Baltes, P. B. \& Baltes, M. M. (1990) Psychological perspectives on successful aging: The model of selective optimization with compensation. In P. B. Baltes, \& M. M. Baltes, Sucessful aging perspectives from the behavioral sciences. (pp. 1-34). Cambridge: Cambridge University Press.

Berri, B., Castro, A., \& Camargo, B. V. (2016). Representações sociais relacionadas às práticas de rejuvenescimento. Psicologia em Pesquisa-UFJF, 10(2), 21-30.

Biasus, F., Demantova, A., \& Camargo, B. V. (2011). Representações sociais do envelhecimento e da sexualidade para pessoas com mais de 50 anos. Temas em Psicologia, 19(1), 319-336.

Brooks, A.T. (2010). Aesthetic anti-ageing surgery and technology: Women's friend or foe? Sociology of Health \& Illness, 32(2), 238-257. https:// doi.org/10.1111/j.1467-9566.2009.01224.x

Caderec, V. (2011). Sexagenários e octagenários diante do envelhecimento do corpo. In M. Goldenberg (Org), Corpo, envelhecimento e felicidade (pp.21-44). Rio de Janeiro, RJ: Cultura Brasileira. 
Camargo, B.V.; Contarello, A.; Wachelke, J.F.R.; Morais, D.X., \& Piccolo, C. (2014). Representações sociais do envelhecimento entre diferentes gerações no Brasil e na Itália. Psicologia em Pesquisa (UFJF), 8(2), 179-188.

Camargo, B. V., Goetz, E. R., Bousfield, A. B. S., \& Justo, A. M. (2011). Representações sociais do corpo: Estética e saúde. Temas em Psicologia, 19(1), 257-268.

Camargo, B. V., \& Justo, A. M. (2013). IRAMUTEQ: Um software gratuito para análise de dados textuais. Temas em Psicologia, 21(2), 513-518.

Camargo, B. V., Justo, A. M., \& Jodelet, D. (2010). Normas, representações sociais e práticas corporais. Interamerican Journal of Psychology, 44(3), 449-457.

Castro, A., Antunes, L., Brito, A. M. M., \& Camargo, B. V. (2016). Representações sociais do envelhecimento e rejuvenescimento para mulheres que adotam práticas de rejuvenescimento. Psico, 47(4), 319-330.

Cruz, R. C., \& Ferreira, M. A. (2011) Um certo jeito de ser velho: representações sociais da velhice por familiares de idosos. Texto \& Contexto: Enfermagem, 20(1), 144-151. https://doi.org/10.1590/S0104-07072011000100017

Dany, L., Urdapilleta, I., \& Lo Monaco, G. (2015). Free association and social representation: some reflections om rank-frequency and importance-frequency methods. Quality \& Quantity: International Journal of Methodology, 49(2), 489-507. https://doi.org/10.1007/s11135-014-0005-z

De Rosa, A,S. (2005). A rede associativa: uma técnica para captar a estrutura, os conteúdos, e os índices de polaridade, neutralidade e estereotipia dos campos semânticos relacionados com as representações sociais. In A. S. P. Moreira, B. V. Camargo, J. C. Jesuíno, \& S. M. Nóbrega, Perspectivas teórico-metodológicas em representações sociais (pp. 61-128, C. C. C. Soares, Trad.). João Pessoa: Ed. Universitária.

Doise, W. (1985). Les représentations sociales: définition d'un concept. Connexions, 45, 243-253.

Ferreira, V.S. (2013). Resgates sociológicos do corpo: esboço de um perscuso conceptual. Análise Social, 48(3), 494-528.

Flament, C. (2001). Estrutura e dinâmica das representações sociais. In D. Jodelet (Org.), As representações sociais (pp. 173-186). Rio de Janeiro, RJ: UERJ.

Flatt, M. A., Settersten Junior, R. A.; Ponsaran, R., \& Fishman, J. R (2013). Are "anti-aging medicine" and "successful aging" two sides of the same coin? Views of anti-aging practitioners. Journals of Gerontology, Series B: Psychological Sciences and Social Sciences, 68(6), 944-955. https://doi.org/10.1093/geronb/gbt086

Instituto Brasileiro de Geografia e Estatística - IBGE. (2016), Síntese de indicadores sociais: uma análise das condições de vida da população brasileira Rio de Janeiro, RJ: o autor.

International Society of Aesthetic Plastic Surgery (2014). ISAPS International Survey on Aesthetic/Cosmetic. Recuperado de: https://www.isaps.org/Media/Default/global-statistics/2015\%20ISAPS\%20Results.pdf

Jodelet, D. (1989). Les representationes sociales. Paris: Press Universitaires de France.

Jovchelovitch , S. (2011) Representações sociais e polifasia cognitiva: Notas sobre a pluralidade e sabedoria da Razão em Psicanálise, sua imagem e seu público. In: A. M. O. Almeida, M. F. Souza, \& Z. A. Trindade (Eds.), Teoria das representações sociais: 50 anos (pp. 159-176). Rio de Janeiro, RJ: Techno Politik.

Magnabosco-Martins, C. R., Camargo, B. V., \& Biasus, F. (2009). Representações sociais do idoso e da velhice para diferentes faixas etárias. Universitas Psychologica, 8(3), 831-847.

Marková, I. (2003). Dialogicality and social representations: The dynamics of mind. NewYork, NY:Cambridge University Press.

Miranda, V. P. N., Filgueiras, J. F., Neves, C. M., Teixeira, P. C., \& Ferreira, M. E. C. (2012). Insatisfação corporal em universitários de diferentes áreas do conhecimento. Jornal Brasileiro de Psiquiatria, 61(1), 25-32. https://doi.org/10.1590/S0047-20852012000100005

Moscovici, S. (1978). A representação social da psicanálise. Rio de Janeiro, RJ: Zahar.

Nóbrega, S. M. (2003). Sobre a teoria das representações sociais. In A. S. P. Moreira, \& J. C. Jesuíno (Orgs), Representações sociais: Teoria e prática. João Pessoa, PB: EDUFPB.

Parales-Quenza, C. J. \& Vizcaíno-Gutiérrez, M. (2007). Las relaciones entre actitudes y representaciones sociales: Elementos para uma integración conceptual. Revista Latinoamericana de Psicología, 39(2), 351-361.

Schneider, R. H. \& Irigaray, T. Q. (2008). O envelhecimento na atualidade: aspectos cronológicos, biológicos, psicológicos e sociais. Estudos de Psicologia, 25(4), 585-593. 
Schulze, C. M. N. (2011). Social representations of ageing shared by different age groups. Temas em Psicologia, 19(1), 43-57

Organização Mundial de Saúde - OMS. (2005). Envelhecimento ativo: Uma política de saúde (S. Gontijo, Trad.). Brasília, DF: Organização Pan-Americana da Saúde.

Torres, T. L. (2010). Pensamento social sobre envelhecimento, idoso e rejuvenescimento para diferentes grupos etários (Tese de Doutorado). Universidade Federal de Santa Catarina, Florianópolis, SC.

Torres, T. L., Camargo, B. V., Bousfield, A. B., \& Silva, A. O. (2015) Representações sociais e crenças normativas sobre envelhecimento. Ciência e Saúde Coletiva, 20(12), 3621-3630. http://doi.org/10.1590/1413812320152012.01042015

Vergès, P. (1992). L'évocation de l'argent: une méthode pour la définition du noyau central de la représentation. Bulletin de Psychologie, 45(405), 203-209.

\section{Adriana de Aguiar}

Doutora em Psicologia pela Universidade Federal deSanta Catarina eatualmentecolaboradora do Laboratório dePsicologia Social da Comunicação e Cognição (LACCOS). É psicóloga da Prefeitura Municipal de Florianópolis, desenvolvendo atividades no âmbito da Política de Assistência Social e com ênfase de atuação no trabalho social com famílias.

E-mail: adrianadeaguiar@yahoo.com.br

\section{Brigido Vizeu Camargo}

Professor Titular do Departamento de Psicologia da Universidade Federal de Santa Catarina

E-mail: brigido.camargo@yahoo.com.br

\section{Andréa Barbará da Silva Bousfield}

Professora Associada do Departamento de Psicologia da Universidade Federal de Santa Catarina

E-mail: andreabs@gmail.com

Endereço para envio de correspondência:

Rua Pintor Eduardo Dias, 738, apto 304

Barreiros - CEP: 88117-013

São José -SC. Brasil.

Recebido 27/10/2017

Aprovado 03/05/2018

Received 10/27/2017

Approved 05/03/2018

Recibido 27/10/2017

Aceptado 03/05/2018

Como citar: Aguiar, A., Camargo, B. V., \& Bousfield, A. B. S. (2018). Envelhecimento e prática de rejuvenescimento: Estudo de representações sociais. Psicologia: Ciência e Profissão, 38(3), 494-506. https://doi.org/10.1590/1982-37030004492017

How to cite: Aguiar, A., Camargo, B. V., \& Bousfield, A. B. S. (2018). Aging and rejuvenation practice: Study of social representations. Psicologia: Ciência e Profissão, 38(3), 494-506. https:// doi.org/10.1590/1982-37030004492017

Cómo citar: Aguiar, A., Camargo, B. V., \& Bousfield, A. B. S. (2018). Envejecimiento y práctica de rejuvenecimiento: Estudio de representaciones sociales. Psicologia: Ciência e Profissão, 38(3), 494-506. https://doi.org/10.1590/1982-37030004492017 